\title{
Functional traits and the growth-mortality trade-off in tropical trees
}

\author{
S. Joseph Wright, ${ }^{1,16}$ Kaoru Kitajima,,${ }^{1,2}$ Nathan J. B. Kraft, ${ }^{3}$ Peter B. Reich, ${ }^{4}$ Ian J. Wright, ${ }^{5}$ \\ Daniel E. Bunker,${ }^{6}$ Richard Condit, ${ }^{1}$ James W. Dalling, ${ }^{1,7}$ Stuart J. Davies, ${ }^{1}$ Sandra Díaz ${ }^{8}$ \\ Bettina M. J. Engelbrecht, ${ }^{1,9}$ Kyle E. Harms, ${ }^{1,10}$ Stephen P. Hubbell, ${ }^{1,11}$ Christian O. Marks, ${ }^{12}$ \\ Maria C. Ruiz-Jaen, ${ }^{13}$ Cristina M. Salvador, ${ }^{14}$ and Amy E. Zanne ${ }^{15}$ \\ ${ }^{1}$ Smithsonian Tropical Research Institute, Apartado 0843-03092, Balboa, Panama \\ ${ }^{2}$ Department of Biology, University of Florida, Gainesville, Florida 32611 USA \\ ${ }^{3}$ Department of Integrative Biology, University of California, Berkeley, California 94720 USA \\ ${ }^{4}$ Department of Forest Resources, University of Minnesota, St. Paul, Minnesota 55108 USA \\ ${ }^{5}$ Department of Biological Sciences, Macquarie University, Sydney, New South Wales 2109 Australia \\ ${ }^{6}$ Department of Biological Sciences, New Jersey Institute of Technology, Newark, New Jersey 07102 USA \\ ${ }^{7}$ Department of Plant Biology, University of Illinois, Champaign-Urbana, Illinois 61801 USA \\ ${ }^{8}$ Instituto Multidisciplinario de Biología Vegetal, Consejo Nacional de Investigaciones Científicas y Técnicas and Facultad de Ciencias \\ Exactas, Físicas y Naturales, Universidad Nacional de Córdoba, Casilla de Correo 495, 5000 Córdoba, Argentina \\ ${ }^{9}$ Department of Plant Ecology, University of Bayreuth, 95440 Bayreuth, Germany \\ ${ }^{10}$ Department of Biological Sciences, Louisiana State University, Baton Rouge, Louisiana 70803 USA \\ ${ }^{11}$ Department of Ecology and Evolutionary Biology, University of California, Los Angeles, California 90095 USA \\ ${ }^{12}$ Department of Ecology, Evolution and Behavior, University of Minnesota, St. Paul, Minnesota 55108 USA \\ ${ }^{13}$ Department of Biology, McGill University, 1205 Dr. Penfield Avenue, Montreal, Quebec H3A 1 B1 Canada \\ ${ }^{14}$ Santa Fe Botanical Garden, P.O. Box 23343, Santa Fe, New Mexico 87502-3343 USA \\ ${ }^{15}$ Department of Biology, One University Boulevard, University of Missouri, St. Louis, Missouri 63121 USA
}

Abstract. A trade-off between growth and mortality rates characterizes tree species in closed canopy forests. This trade-off is maintained by inherent differences among species and spatial variation in light availability caused by canopy-opening disturbances. We evaluated conditions under which the trade-off is expressed and relationships with four key functional traits for 103 tree species from Barro Colorado Island, Panama. The trade-off is strongest for saplings for growth rates of the fastest growing individuals and mortality rates of the slowest growing individuals $\left(r^{2}=0.69\right)$, intermediate for saplings for average growth rates and overall mortality rates $\left(r^{2}=0.46\right)$, and much weaker for large trees $\left(r^{2} \leq 0.10\right)$. This parallels likely levels of spatial variation in light availability, which is greatest for fast- vs. slow-growing saplings and least for large trees with foliage in the forest canopy. Inherent attributes of species contributing to the trade-off include abilities to disperse, acquire resources, grow rapidly, and tolerate shade and other stresses. There is growing interest in the possibility that functional traits might provide insight into such ecological differences and a growing consensus that seed mass (SM), leaf mass per area (LMA), wood density (WD), and maximum height $\left(H_{\max }\right)$ are key traits among forest trees. Seed mass, LMA, WD, and $H_{\max }$ are predicted to be small for light-demanding species with rapid growth and mortality and large for shade-tolerant species with slow growth and mortality. Six of these trait-demographic rate predictions were realized for saplings; however, with the exception of WD, the relationships were weak $\left(r^{2}<0.1\right.$ for three and $r^{2}<0.2$ for five of the six remaining relationships). The four traits together explained $43-44 \%$ of interspecific variation in species positions on the growth-mortality trade-off; however, WD alone accounted for $>80 \%$ of the explained variation and, after WD was included, LMA and $H_{\max }$ made insignificant contributions. Virtually the full range of values of SM, LMA, and $H_{\max }$ occurred at all positions on the growth-mortality trade-off. Although WD provides a promising start, a successful traitbased ecology of tropical forest trees will require consideration of additional traits.

Key words: Barro Colorado Island, Panama; growth rates; leaf mass per area; maximum height; maximum size; mortality rates; seed mass; seed size; wood density.

\section{INTRODUCTION}

A demographic trade-off between rates of growth vs. mortality is perhaps the best-established axis of life

Manuscript received 19 December 2009; revised 29 March 2010; accepted 9 April 2010; final version received 12 May 2010. Corresponding Editor: F. He.

${ }^{16}$ E-mail: wrightj@si.edu history variation among tree species in closed-canopy forests (Grubb 1977, Hubbell and Foster 1992, Pacala et al. 1996, Wright et al. 2003, Kitajima and Poorter 2008). The trade-off is maintained by inherent differences among species in combination with spatial variation in light availability caused by treefalls and other canopyopening disturbances. At one extreme, light-demanding species readily colonize forest gaps and grow quickly if 
light levels remain high or die quickly if overtopped and shaded. At the other extreme, shade-tolerant species survive canopy-opening events as juveniles, have limited potential growth rates, and are overtopped by fastgrowing, light-demanding species with limited impact on mortality risk. Thus, the trade-off concerns growth rates realized when resource availability is high (and other stresses are absent, henceforth "favorable conditions") vs. mortality rates realized when resource availability is low (or other stresses are present, henceforth "unfavorable conditions").

The inherent differences among species that contribute to this growth-mortality trade-off concern the abilities to disperse, to acquire resources and to grow rapidly, and to tolerate shade and other stresses. There is growing interest in the possibility that functional traits provide insight into these and other ecological differences among species, where functional traits refer to well-defined, measurable properties of organisms that strongly influence or are strongly coordinated with ecological performance (Lavorel and Garnier 2002, Reich et al. 2003, McGill et al. 2006, Westoby and Wright 2006). There is also a growing consensus that wood density (WD), seed mass (SM), leaf mass per area (LMA), and maximum adult height $\left(H_{\max }\right)$ are key functional traits among forest trees providing insight into biogeochemical cycles (WD and LMA); life history variation (WD, SM, and LMA); and the ability to disperse to new sites (SM), acquire resources (LMA), grow quickly (WD and LMA), compete with neighbors (LMA, SM, and $H_{\max }$ ), and tolerate pests and other hazards (WD, SM, and LMA) (Hodgson et al. 1999, Weiher et al. 1999, Lavorel and Garnier 2002, Westoby et al. 2002, Cornelissen et al. 2003, Reich et al. 2003, Díaz et al. 2004, Westoby and Wright 2006, Wright et al. 2007, Chave et al. 2009).

The hundreds of sympatric tree species present in many tropical forests present an opportunity to evaluate hypothesized relationships between functional traits and life history variation as well as a challenge. The challenge concerns the initial documentation of life history variation. It is impractical to assess environmental conditions for enough individuals to evaluate a resource-dependent, growth-mortality trade-off for hundreds of species, particularly when most of those species are exceedingly rare. Instead, average growth rates and overall mortality rates are evaluated without reference to resource availability with the result that average growth-overall mortality relationships are often insignificant (Condit et al. 2006, Poorter et al. 2008). Here we evaluate the growth-mortality trade-off for growth rates of successively smaller subsets of the fastest growing individuals and mortality rates of successively smaller subsets of the individuals that grew most slowly in the previous census for six censuses of 368122 trees on Barro Colorado Island (BCI), Panama. We predict that the growth-mortality trade-off will be stronger for these successively smaller subsets of individuals because growth rates of the fastest growing individuals approximate potential growth rates under favorable conditions while mortality rates of the slowest growing individuals approximate the ability to survive unfavorable conditions.

After documenting each species position on the growth-mortality trade-off, we evaluated relationships with functional traits. Classical life history theory and common garden experiments from a wide range of vegetation types indicate that small SM, LMA, and WD characterize light-demanding or more generally highresource-consuming species and that the opposite trait values characterize shade-tolerant or more generally stress-tolerant species (Grubb 1977, Kitajima 1994, Grime et al. 1997, Hodgson et al. 1999, Westoby et al. 2002, Cornelissen et al. 2003, Reich et al. 2003, Westoby and Wright 2006, Poorter et al. 2008, Chave et al. 2009, Muller-Landau 2010). Poorter et al. (2008) also hypothesized that growth and mortality rates should be inversely related to $H_{\max }$ because species adapted to ephemeral forest gaps grow fast and should reproduce quickly at small adult sizes while species adapted to closed-canopy conditions grow slowly and should be tall and long-lived. Thus, we evaluated the predictions that growth rates, mortality rates, and position on the growth-mortality trade-off are inversely related to SM, LMA, WD, and $H_{\text {max }}$.

Most tropical forest plant species are rare ecological ciphers, and the possibility that their life histories might be revealed by measurements of a few key functional traits has been greeted enthusiastically. Increasingly, studies of tropical trees evaluate relationships between mean growth rates and/or overall mortality rates and one or two functional traits, most frequently WD (reviewed in Discussion). Poorter et al. (2008) provide an exception in that they evaluated the same four functional traits considered here. In pairwise analyses, the individual traits consistently explained a small proportion of interspecific variation in mean growth and overall mortality rates of large trees from five Neotropical forests $\left(r^{2}<0.1\right.$ for $60 \%$ and $<0.2$ for $82.5 \%$ of comparisons). Collectively the four functional traits fared little better in multiple regression analyses explaining just $11 \%$ and $29 \%$ of interspecific variation in growth and mortality rates, respectively. Poorter et al. (2008) speculated that trait-performance relationships would be stronger for saplings than for the large trees that they considered. We evaluated the growth-mortality trade-off and relationships with functional traits for saplings as well as for large trees, for growth rates of successively smaller subsets of the most rapidly growing individuals to approximate potential growth rates under favorable conditions, and for mortality rates of successively smaller subsets of the most slowly growing individuals to approximate the potential to survive unfavorable conditions. Finally, we explored the range of trait combinations that characterize species at all points along the growth-mortality trade-off manifold. 


\section{Methods}

All free-standing woody plants with diameter at breast height $(\mathrm{dbh}) \geq 1 \mathrm{~cm}$ were measured in a 50 -ha plot on BCI in 1982, 1985, 1990, 1995, 2000, and 2005 (Hubbell and Foster 1992, Condit et al. 2006). Relative growth rates (RGR) equaled $\ln \left(\mathrm{dbh}_{\mathrm{f}} / \mathrm{dbh}_{\mathrm{i}}\right) /\left[\left(t_{\mathrm{f}}-t_{\mathrm{i}}\right) /\right.$ 365 ], where $t$ is day of century and the subscripts $f$ and $\mathrm{i}$ are final and initial values, respectively. Mortality rates $(M)$ equaled $100 \times\left[1-\left(N_{\mathrm{f}} / N_{\mathrm{i}}\right)\right]$ for $N_{\mathrm{i}}$ initial individuals and $N_{\mathrm{f}}$ survivors. Growth rates under favorable conditions equaled 95th percentile relative growth rates $\left(\mathrm{RGR}_{95}\right)$. Mortality rates under unfavorable conditions were calculated for the $25 \%$ of individuals of each species with the smallest RGR in the previous census interval $\left(M_{25}\right)$. The first census interval lacked prior estimates of RGR so was not used to estimate mortality. We did not annualize mortality rates because subsequent census intervals were fixed at $5 \mathrm{yr}$. We also calculated mean RGR, $\mathrm{RGR}_{90}$, overall mortality, and $M_{50}$. To limit sapling analyses to sterile individuals, we excluded individuals with $\mathrm{dbh}_{\mathrm{i}} \geq 5 \mathrm{~cm}$ and species with $H_{\max }<5$ m. We also calculated mean RGR, RGR ${ }_{90}, \mathrm{RGR}_{95}$, overall mortality, $M_{50}$, and $M_{25}$ for trees of the same large size $\left(10 \mathrm{~cm} \leq \mathrm{dbh}_{\mathrm{i}}<50 \mathrm{~cm}\right)$ evaluated by Poorter et al. (2008). We excluded palms because most palm species lack dbh growth, species with fewer than 100 observations to calculate $M_{25}$, RGR values for trees whose measurement height for dbh changed between censuses due to the growth of buttresses, and sapling RGR values involving the 1982 and 1985 censuses because $\mathrm{dbh}$ values $<5 \mathrm{~cm}$ were rounded down to the nearest $5 \mathrm{~mm}$ in these first two censuses.

We measured SM, WD, LMA, and $H_{\max }$ following Cornelissen et al. (2003) with two exceptions. First, our SM refers to endosperm and embryo only while Cornelissen et al. (2003) recommend using diaspores. Our SM controls interspecific variation in allocation to endocarps and testa, which averaged $34 \% \pm 21 \%$ (mean $\pm 1 \mathrm{SD}$ ) of diaspore mass among BCI species. The second difference concerned LMA. We measured LMA for leaves taken from the tallest foliage of six of the smallest individuals of each species in the 50-ha plot. We did not use the sun-exposed leaves recommended by Cornelissen et al. (2003) because most BCI tree species reproduce in the understory and lack sun-exposed leaves (King et al. 2006b). We did, however, measure LMA for leaves from the tallest foliage of the six largest individuals of each species. The relationship between LMA for sun-exposed leaves and for the shaded understory leaves used here was strong $\left(r^{2}=0.57, P<\right.$ $0.001)$ for the 25 species with LMA determinations for five or more individuals in both light habitats. Maximum height, $H_{\max }$, equaled the mean height of the six largest (by dbh) individuals of each species in the 50-ha plot and a nearby $(5 \mathrm{~km})$ 38.4-ha plot (King et al. 2006b). To assess WD, we cored trees located within 15 $\mathrm{km}$ of BCI because increment borers are prohibited on BCI. We broke cores into pieces $<5 \mathrm{~cm}$ long, calculated the specific gravity of each piece as oven-dried mass $\left(100^{\circ} \mathrm{C}\right)$ divided by fresh volume (measured by water displacement), and estimated WD as an area-weighted average, where area refers to the annulus represented by each piece, assuming a circular trunk.

We performed correlation analyses to evaluate the growth-mortality trade-off and pairwise relationships between $\mathrm{RGR}_{95}, M_{25}, H_{\max }$, LMA, WD, and SM for saplings using species values and phylogenetically independent contrasts (PICs) (Felsenstein 1985). We repeated the species-level correlation analyses for large trees to enable direct comparison with Poorter et al. (2008). We performed $z$ tests to evaluate the hypothesis that correlation coefficients were equal.

We performed two principal components analyses. The first was for the $\mathrm{RGR}_{95}-M_{25}$ relationship. In subsequent analyses, species positions on the growthmortality trade-off equaled their factor score on the first principal component of the $\mathrm{RGR}_{95}-M_{25}$ relationship. The second principal component analysis evaluated relationships among the four functional traits.

We performed three multiple regression analyses to evaluate relationships between traits (independent variables) and $\mathrm{RGR}_{95}, M_{25}$, and position on the growthmortality trade-off (three dependent variables) for saplings. The multiple regression analyses were repeated for species values and PICs. Multiple regression diagnostics were evaluated for collinearity, which occurs when condition indices (largest eigenvalue divided by each smaller eigenvalue) are $>30$ or between 15 and 30 if variance proportions are large for two or more independent variables (Belsley et al. 1980). Collinearity was absent. The largest condition index of 17.0 occurred in a model that included all four traits with just one having a largish variance proportion. All condition indices were $<15$ in models that excluded insignificant traits. The traits were effectively independent for the purposes of multiple regression analysis despite weak but significant correlations for two of the six trait pairs (see Results: Functional traits).

We performed a discriminant analysis to evaluate a standard practice in which tropical tree species are placed into light-demanding, shade-tolerant, and often one intermediate category. To approximate this practice, we divided ranked factor scores from the first principal component of the $\mathrm{RGR}_{95}-M_{25}$ relationship into thirds to represent light-demanding, intermediate, and shadetolerant species (Fig. 1). The discriminant analysis then determined the proportion of species classified into these three categories correctly given species-specific values of SM, LMA, WD, and $H_{\text {max }}$. The discriminant analysis is technically inappropriate because $\mathrm{RGR}_{95}-M_{25}$ factor scores are continuous rather than categorical. We included the discriminant analysis here because it is the most direct method to evaluate the standard practice of categorizing tropical trees by their supposed life histories. 
We calculated PICs for a DNA barcode phylogeny based on three plastid markers $(r b c L, m a t K$, and $\operatorname{trnH}$ $p s b A$ ) for 281 species from the BCI 50 -ha plot (Kress et al. 2009). The barcode phylogeny and the angiosperm phylogeny group current hypothesis resolved $97.5 \%$ vs. just $48.4 \%$ of nodes among these 281 species, respectively (Kress et al. 2009). We used the analysis of traits (AOT) module of the program Phylocom (Webb et al. 2008) to calculate contrasts. At polytomies, one trait was selected arbitrarily, its median value was used to divide the taxa involved in the polytomy into two groups, and the mean trait values for the two groups were used to calculate contrasts, as recommended by Pagel (1992).

All other analyses were performed with SYSTAT 11.0 (Richmond, California, USA) on untransformed values of $H_{\max }, \mathrm{LMA}, \mathrm{WD}$, and mortality rates and logtransformed values of SM and growth rates. We forced regressions of PIC data through the origin. This is necessary because the direction of subtraction is arbitrary when contrasts are calculated. A contrast with positive divergences in two traits would have negative divergences in both traits had the subtractions been performed the other way around. Due to this symmetry, regressions of contrast data have no intercept term; they are "forced" through the origin (Garland et al. 1992).

\section{RESUlTS}

\section{Growth-mortality trade-off in saplings}

We calculated mean RGR, $\mathrm{RGR}_{90}, \mathrm{RGR}_{95}$, overall mortality, $M_{50}$, and $M_{25}$ for saplings of 103 species with $H_{\max }>5 \mathrm{~m}$ (see Supplement). Interspecific standard deviations of growth and mortality rates and the strength of the growth-mortality trade-off increased consistently as RGR was calculated for ever-smaller subsets of the fastest growing individuals and as mortality rates were calculated for ever-smaller subsets of the slowest growing individuals. The trade-off between $\mathrm{RGR}_{95}$ and $M_{25}\left(r^{2}=0.691, P<0.001\right.$; Fig. $1)$ was significantly stronger $(z=2.57, P<0.01)$ than the trade-off between mean RGR and overall mortality (not shown; $\left.r^{2}=0.462, P<0.001\right)$. RGR 95 and $M_{25}$ varied nearly fivefold $\left(0.073-0.34 \mathrm{~cm} \cdot \mathrm{cm}^{-1} \cdot \mathrm{yr}^{-1}\right)$ and 15 -fold (5.5-87.5\% per 5 yr) among species, respectively.

\section{Growth-mortality trade-off in large trees}

We calculated mean RGR, $\mathrm{RGR}_{90}, \mathrm{RGR}_{95}$, overall mortality, $M_{50}$, and $M_{25}$ for large trees of 95 species (see Supplement). Interspecific standard deviations of growth and mortality rates increased consistently as RGR was calculated for ever-smaller subsets of the fastest growing individuals and as mortality rates were calculated for ever-smaller subsets of the slowest growing individuals. The strength of the growthmortality trade-off was invariably modest. Coefficients of determination $\left(r^{2}\right.$ values) varied from 0.01 for the relationship between mean RGR and overall mortality $(P=0.21)$ to a maximum of just 0.10 for the relationship between $\mathrm{RGR}_{90}$ and $M_{25}(P=0.006)$. This maximal

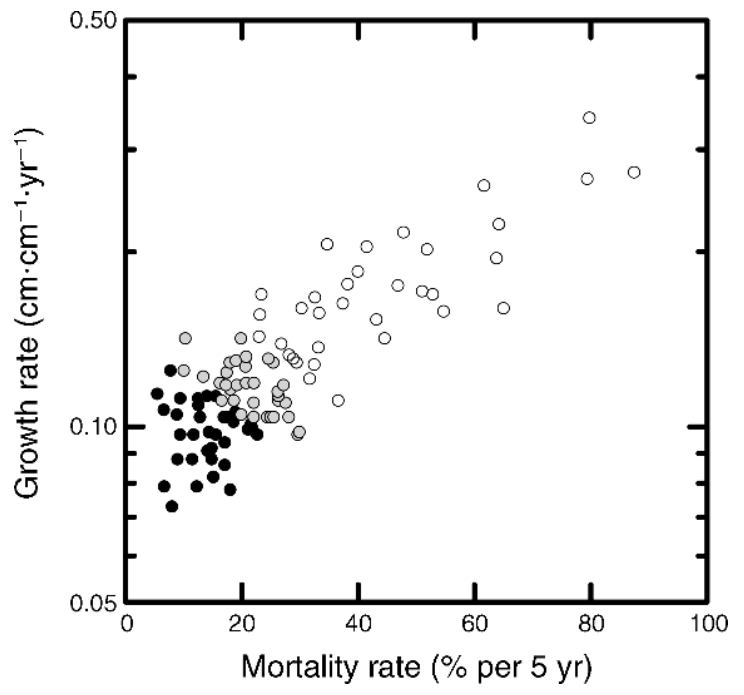

FIG. 1. The growth-mortality trade-off for saplings expressed as the 95th percentile relative growth rate $\left(\mathrm{RGR}_{95}\right)$ vs. the mortality rate of the slowest growing $25 \%$ of individuals $\left(M_{25}\right)$ for 103 tree species with mean maximum height $>5 \mathrm{~m}$ on Barro Colorado Island, Panama. Open, shaded, and solid symbols represent species with factor scores in the top, middle, and bottom thirds, respectively, for the first principal component of the $\mathrm{RGR}_{95}-M_{25}$ trade-off. Minimum sample sizes are 100 trees/species for $M_{25}$ and 333 trees/species for RGR 95 .

difference in $r^{2}$ values was insignificant $(z=-1.24, P=$ $0.21)$.

\section{Functional traits}

We determined $H_{\max }$, LMA, WD, and SM for 103 , 103,100 , and 76 of the 103 sapling species, respectively (see Supplement). Maximum height varied sixfold (6.1$40.4 \mathrm{~m})$, LMA fourfold $\left(27-105 \mathrm{~g} / \mathrm{m}^{2}\right)$, WD nearly threefold $\left(0.31-0.84 \mathrm{~g} / \mathrm{cm}^{3}\right)$, and SM by six orders of magnitude (0.00005-22 g). Small coefficients of determination characterized pairwise relationships among traits (Fig. 2); however, the LMA-WD (Fig. 2E) and LMA-SM (Fig. 2F) relationships were significant despite $r^{2}$ values of just 0.13 and 0.12 (Bonferronicorrected $P$ values $=0.001$ and 0.014 , respectively). Coefficients of determination were remarkably similar for PIC and species-level analyses of trait-trait relationships (not shown).

Two significant principal components explained $39.6 \%$ and $31.7 \%$ of variation among traits for the 75 sapling species with all four traits measured. The first component had large positive loadings for WD and LMA and the second had large positive loadings for $H_{\max }$ and SM (Fig. 3).

\section{Functional traits and demographic rates among saplings}

In pairwise analyses, the direction of seven of the eight predictions (see Introduction) was confirmed although coefficients of determination were often small. Wood density was the strongest predictor of $\mathrm{RGR}_{95}$ and $M_{25}$, 


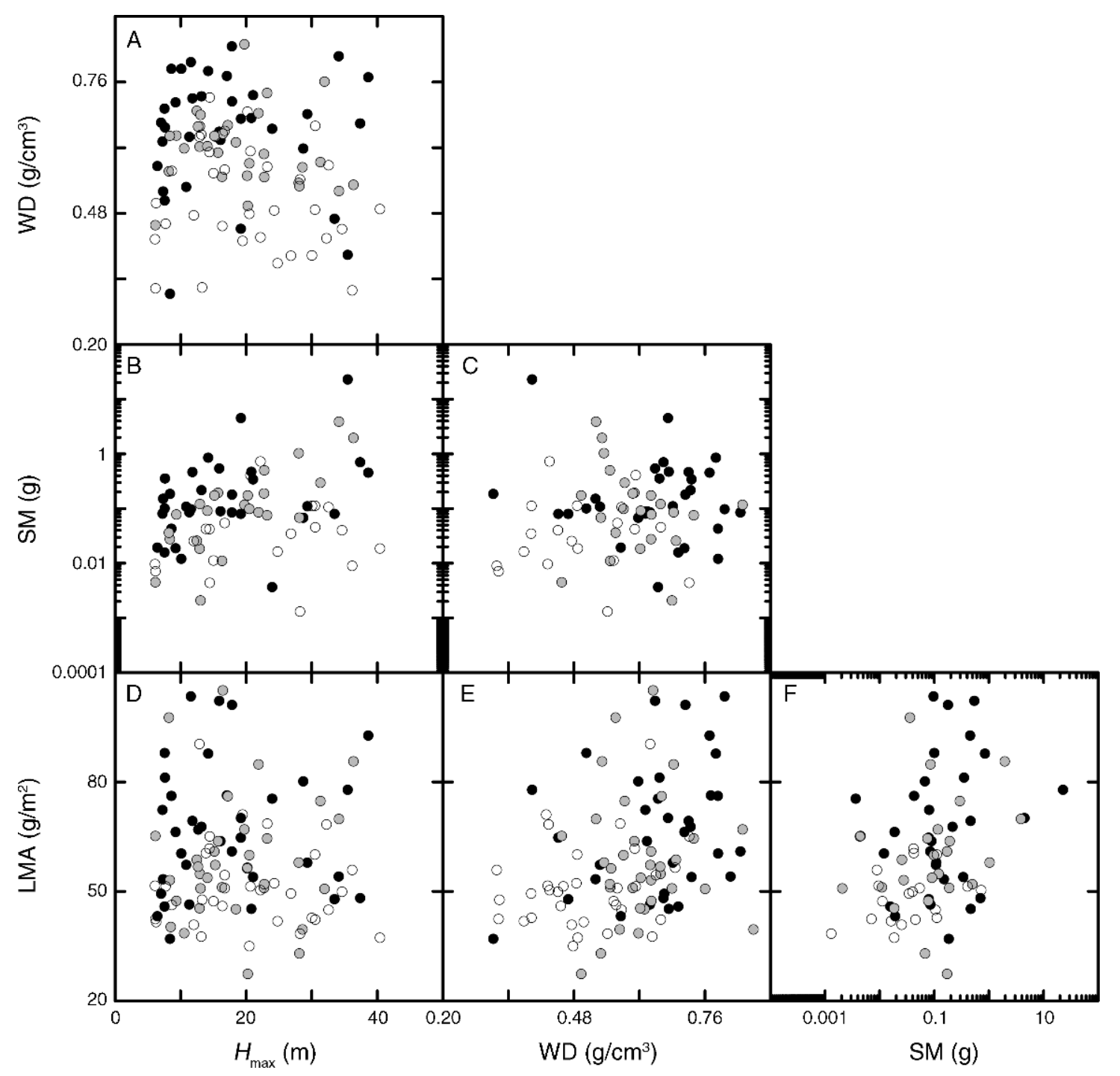

FIG. 2. Pairwise relationships among maximum height $\left(H_{\max }\right)$, wood density (WD), seed mass (SM), and leaf mass per area (LMA) for trees on Barro Colorado Island, Panama. Open, shaded, and solid symbols are as in Fig. 1 and illustrate the wide range of trait values found at all positions on the growth-mortality trade-off. Coefficients of determination $\left(r^{2}\right)$ are (A) 0.017 , (B) 0.052 , (C) 0.0056, (D) 0.00068, (E) 0.13, and (F) 0.12.

explaining $29-41 \%$ of interspecific variation in specieslevel and PIC analyses (Table 1). Leaf mass per area was the next strongest single predictor, explaining $18-24 \%$ of interspecific variation in species-level analyses and 9$13 \%$ in PIC analyses. Seed mass and $H_{\max }$ were considerably weaker predictors of both $\mathrm{RGR}_{95}$ and $M_{25}$. The $\mathrm{RGR}_{95}-H_{\max }$ relationship was positive, not negative as predicted, but with a coefficient of determination of just 0.076 (Table 1A). Seed mass explained $15 \%$ of interspecific variation in $M_{25}$ in the species-level analysis (Table 1A); however, this fell to $4.1 \%$ in the PIC analysis (Table 1B). In contrast, coefficients of determination were similar for PIC and species-level analyses for the seven remaining pairwise relationships between traits and demographic rates (Table 1).
In multiple regression analyses, the four traits collectively explained $39-43 \%$ of interspecific variation in $\mathrm{RGR}_{95}, M_{25}$, and position on the growth-mortality trade-off in species-level analyses and $34-50 \%$ in PIC analyses (Table 1). Wood density accounted for 66-89\% of the total variation explained by significant traits across the six multiple regression analyses. Leaf mass per area explained significant additional variation after WD in just two of the six multiple regressions despite being a consistently strong predictor of demographic rates in pairwise analyses. In contrast, SM explained significant additional variation after WD in four of the six multiple regressions despite weak relationships with vital rates in three of the four pairwise analyses. Once again, $H_{\max }$ contributed little. 


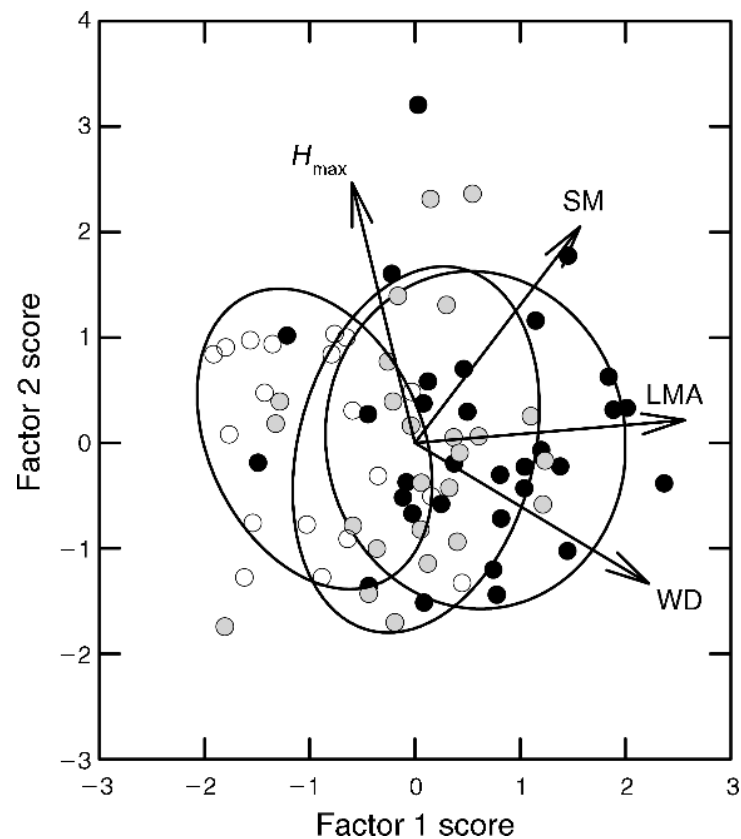

FIG. 3. Principal components analysis for maximum height $\left(H_{\max }\right)$, seed mass $(\mathrm{SM})$, leaf mass per area (LMA), and wood density (WD) for tropical trees on Barro Colorado Island, Panama. Arrows represent principal components loadings. Open, shaded, and solid symbols are as in Fig. 1 and illustrate the wide range of trait values found at all positions on the growthmortality trade-off. The broadly overlapping ellipses represent one sample standard deviation for species in the three inferred life history categories: light-demanding (open symbols), intermediate (gray symbols), and shade-tolerant (black symbols) species.
The discriminant analysis classified 58\%, 32\%, and $68 \%$ of shade-tolerant, intermediate, and light-demanding species correctly where these species had small, intermediate, and large factor scores on the first principal component of the $\mathrm{RGR}_{95}-M_{25}$ relationship, respectively (Fig. 3). This compares with 33\% correct classification expected by chance given three categories. The classification of BCI trees species into lightdemanding, intermediate, and shade-tolerant categories is fraught with error even when informed by extensive trait data.

Functional traits and demographic rates among large trees

Table 2 contrasts three estimates of the strength of relationships between functional traits and demographic rates for large BCI trees $\left(10 \mathrm{~cm} \leq \mathrm{dbh}_{\mathrm{i}}<50 \mathrm{~cm}\right)$. Coefficients of determination for mean RGR and overall mortality are rather similar in Poorter et al. (2008) and in this study, with the notable exception of the SM-mortality relationship (see Discussion: Seed mass). Coefficients of determination for $M_{25}$ and $\mathrm{RGR}_{95}$ tend to be larger than those obtained using mean RGR and overall mortality; however, the differences are not significant.

Relationships between functional traits and $M_{25}$ and RGR $_{95}$ are evaluated for saplings in the second and third columns of Table 1A and for large trees in the final two columns of Table 2. The correlation coefficients are marginally significantly larger for saplings for the WD$M_{25}$, LMA- $M_{25}$, and LMA-RGR ${ }_{95}$ relationships and for large trees for the $H_{\max }-\mathrm{RGR}_{95}$ relationship (abs $|z|$ $>1.7, P<0.10)$.

TABle 1. Coefficients of determination $\left(r^{2}\right)$ for pairwise correlation and multiple regression analyses of relationships between key functional traits, growth rates, mortality rates, and position on the growth-mortality trade-off for tree saplings from Barro Colorado Island, Panama: (A) species values and (B) phylogenetically independent contrasts.

\begin{tabular}{|c|c|c|c|c|c|}
\hline \multirow[b]{3}{*}{ Independent variable } & \multirow{2}{*}{\multicolumn{2}{|c|}{ Correlation analyses }} & \multicolumn{3}{|c|}{ Multiple regression analyses } \\
\hline & & & \multirow[b]{2}{*}{$M_{25}$} & \multirow[b]{2}{*}{$\mathrm{RGR}_{95}$} & \multirow{2}{*}{$\begin{array}{l}\mathrm{RGR}_{95}-M_{25} \\
\text { factor score }\end{array}$} \\
\hline & $M_{25}$ & $\mathrm{RGR}_{95}$ & & & \\
\hline \multicolumn{6}{|l|}{ A) Species values } \\
\hline WD & $-0.31 * * *$ & $-0.33 * * *$ & $-0.31 * * *$ & $-0.33^{* * *}$ & $-0.36^{* * *}$ \\
\hline SM & $-0.15^{* *}$ & $-0.066^{*}$ & $-0.12 * * *$ & $\cdots$ & $-0.087 * *$ \\
\hline LMA & $-0.17 * * *$ & $-0.24 * * *$ & $\cdots$ & $-0.076^{* *}$ & $\cdots$ \\
\hline$H_{\max }$ & 0.0004 & $+0.076^{*}$ & $\cdots$ & $\cdots$ & $\cdots$ \\
\hline Adjusted multiple $R^{2}$ & $\cdots$ & $\cdots$ & 0.41 & 0.39 & 0.43 \\
\hline \multicolumn{6}{|c|}{ B) Phylogenetically independent contrasts } \\
\hline WD & $-0.29 * * *$ & $-0.41 * * *$ & $-0.29 * * *$ & $-0.40^{* * *}$ & $-0.40^{* * *}$ \\
\hline SM & 0.041 & $-0.067 *$ & & $-0.055^{* *}$ & $-0.050^{*}$ \\
\hline LMA & $-0.090^{* *}$ & $-0.13^{* * *}$ & $-0.060 *$ & $\ldots$ & $\ldots$ \\
\hline$H_{\max }$ & 0.004 & $+0.056^{*}$ & $\cdots$ & $+0.062 * *$ & $\cdots$ \\
\hline Adjusted multiple $R^{2}$ & $\cdots$ & $\cdots$ & 0.34 & 0.50 & 0.44 \\
\hline
\end{tabular}

Notes: Significant entries are preceded by the sign of the relationship. For multiple regressions, only significant terms in models with all four independent variables are presented. The independent variables are wood density (WD), seed dry mass (SM), maximum height $\left(H_{\max }\right)$, and leaf mass per area (LMA). The dependent variables are mortality rates of the slowest-growing $25 \%$ of saplings $\left(M_{25}\right)$, 95th percentile sapling growth rates $\left(\mathrm{RGR}_{95}\right)$, and factor scores on the first principal component for the $\mathrm{RGR}_{95}-M_{25}$ relationship. Multiple regression analyses included all independent variables; entries are provided for significant independent variables only. The final row provides the adjusted multiple $R^{2}$ for the multiple regression models.

$* P<0.05 ; * * P<0.01 ; * * * P<0.001$. 
TABLE 2. Relationships between functional traits and growth and mortality rates for large trees $(10 \mathrm{~cm} \leq \mathrm{dbh}<50 \mathrm{~cm})$ from Barro Colorado Island, Panama.

\begin{tabular}{|c|c|c|c|c|c|c|}
\hline \multirow[b]{2}{*}{$\begin{array}{l}\text { Demographic } \\
\text { variable }\end{array}$} & \multicolumn{2}{|c|}{ Poorter et al. (2008) } & \multicolumn{4}{|c|}{ This study ( $N=67$ species $)$} \\
\hline & $\begin{array}{l}\text { Overall } \\
\text { mortality }\end{array}$ & $\begin{array}{l}\text { Mean } \\
\text { RGR }\end{array}$ & $\begin{array}{l}\text { Overall } \\
\text { mortality }\end{array}$ & $\begin{array}{l}\text { Mean } \\
\text { RGR }\end{array}$ & $M_{25}$ & $\mathrm{RGR}_{95}$ \\
\hline $\mathrm{W}$ & & -0.1 & $\overline{-0.0}$ & $* * *$ & $-0.081 *$ & $-0.26 * * *$ \\
\hline SM & $-0.13 * *$ & 0.0004 & 0.0036 & $-0.072 *$ & 0.014 & $-0.094 *$ \\
\hline LMA & 0.020 & 0.056 & 0.020 & 0.0079 & 0.017 & $-0.067 *$ \\
\hline$H_{\max }$ & 0.026 & $+0.12 * *$ & 0.019 & $+0.17 * * *$ & 0.0029 & $+0.28 * * *$ \\
\hline
\end{tabular}

Notes: Entries are coefficients of determination $\left(r^{2}\right)$ preceded by the sign of significant relationships. The independent variables are wood density (WD), seed dry mass (SM), leaf mass per area (LMA), and maximum height $\left(H_{\max }\right)$. The dependent variables are overall mortality rates, average relative growth rates (RGR), the mortality rate of the slowest growing $25 \%$ of individuals $\left(M_{25}\right)$ and 95th percentile RGR $\left(\mathrm{RGR}_{95}\right)$.

$* P<0.05$; ** $P<0.01 ; * * * P<0.001$.

\section{DisCUSSION}

A trade-off between growth rates and mortality rates is perhaps the best established axis of life history variation among forest tree species (Grubb 1977, Hubbell and Foster 1992, Pacala et al. 1996, Wright et al. 2003, Kitajima and Poorter 2008). We have shown that the strength of this trade-off depends strongly on the plants evaluated. The trade-off is strongest for saplings when growth rates of the fastest growing individuals and mortality rates of the slowest growing individuals are evaluated ( $r^{2}=0.69$; Fig. 1), intermediate for saplings when average growth rates and overall mortality rates are evaluated $\left(r^{2}=0.46\right)$, and much weaker for large trees $\left(r^{2} \leq 0.10\right)$. The environmental conditions responsible for the growth-mortality trade-off in closedcanopy forests are well aligned with the observed differences in the strength of the trade-off.

Light availability is a key environmental variable in closed-canopy forests because light limits photosynthetic carbon gain, which in turn influences growth and mortality. Light availability varies spatially with treefalls and other canopy-opening disturbances. The degree of spatial variation in light availability declines predictably with height above the forest floor as background light levels increase (Yoda 1974). The foliage of saplings is in the understory where light availability is most variable. The foliage of large trees is in the subcanopy and canopy where spatial variation in light availability is greatly reduced. Thus, interspecific variation in light availability is potentially greater for saplings than for large trees. In addition, mean growth and overall mortality rates integrate performance over the entire range of light levels encountered by each species. In contrast, the fastest and slowest growing individuals tend to be associated with high and low light levels, respectively, thereby capturing spatial variation in light availability. Thus, the growth-mortality trade-off is expressed more strongly among saplings than among large trees and when growth rates of the fastest growing individuals are contrasted with mortality rates of the slowest growing individuals.

\section{Relationships between functional traits and demographic rates}

Relationships between functional traits and demographic rates were generally in the direction predicted (see Introduction for predictions), tended to be stronger for saplings than for large trees, but tended to have modest $r^{2}$ values even for saplings (Tables 1 and 2). We used unprecedented sample sizes to quantify demographic rates (e.g., $\geq 400$ and 333 trials for sapling mortality and RGR, respectively), measured $H_{\max }$ directly rather than using qualitative values as is often done, and measured traits for each species on BCI (or within $15 \mathrm{~km}$ for WD) rather than using values from remote sites, thereby avoiding geographic variation (Patiño et al. 2009). For these reasons, the modest $r^{2}$ values that characterized trait-demography relationships are likely to be real.

These trait-demography relationships might be inherently weak for at least three reasons. First, each functional trait might influence demographic rates at a different life stage; we might be evaluating an important trait but at the wrong life stage (Grime et al. 1997, Poorter 2007). Second, functional traits might be involved in multiple trade-offs with contradictory effects on demographic rates (Marks and Lechowicz 2006). Third, SM, LMA, WD, and $H_{\max }$ are "soft" in contrast to "hard" traits. "Hard" or fundamental traits are mechanistically linked to performance but are often difficult to measure and are replaced by easier-tomeasure "soft" traits that experience suggests serve as adequate proxies (Hodgson et al. 1999, Weiher et al. 1999, Lavorel and Garnier 2002, Cornelissen et al. 2003, Reich et al. 2003). Here we evaluate these three possible explanations of weak trait-demography relationships for each trait.

\section{Wood density}

The strongest trait-demography relationships were consistently for WD. Growth rates and WD are negatively related at all stages of development after germination (Kitajima 1994, Muller-Landau 2004, 
Poorter et al. 2008, Alvarez-Clare and Kitajima 2009, Chave et al. 2009). Possible mechanisms include greater construction costs as well as lower xylem conductance and hence lower photosynthetic potentials with greater WD (reviewed by Chave et al. 2009).

The relationship between WD and mortality was substantially stronger for saplings $\left(r^{2}=0.31\right)$ than for large trees $\left(r^{2}=0.081\right)$ in this study. We hypothesize that this reflects coordination between WD and shade tolerance (reviewed by Chave et al. 2009) combined with relief from light limitation and associated mortality among large trees whose foliage reaches into the canopy or subcanopy. Possible mechanisms relating WD and mortality include greater structural strength as well as greater pest resistance with greater WD (reviewed by Chave et al. 2009). In sum, WD contributed directly to the growth-mortality trade-off observed among saplings through relatively strong negative correlations with both growth and mortality rates.

\section{Leaf mass per area}

Leaf mass per area was the second best predictor of growth and mortality rates of saplings (Table 1). Leaf mass per area was also negatively correlated with both growth and mortality rates and might be expected to contribute directly to the growth-mortality trade-off; however, this was not the case in multiple regression analyses (Table 1). There was a weak positive relationship between LMA and WD $\left(r^{2}=0.13\right.$; Fig. 2E). Much of the variation in demographic rates explained by LMA in pairwise analyses was apparently explained by WD in multiple regression analyses.

Leaf mass per area illustrates two of the reasons that trait-demography relationships might be inherently weak at the sapling stage. The first is ontogenetic. There is a strong negative relationship between LMA and early seedling growth rates; however, direct limitation of growth rates by large LMA decreases in importance as trees mature and leaf number increases (Lambers and Poorter 1992, Reich et al. 1992, Poorter et al. 2009). In this study, the LMA-RGR ${ }_{95}$ relationship was substantially stronger for saplings $\left(r^{2}=0.24\right)$ than for large trees $\left(r^{2}=0.067\right)$. The LMA- $M_{25}$ relationship was also substantially stronger for saplings $\left(r^{2}=0.18\right)$ than for large trees $\left(r^{2}=0.017\right)$. This is consistent with ongoing declines in the importance of LMA for demographic rates as trees mature.

The second reason LMA-demography relationships might be inherently weak is because LMA is a "soft" trait. Other leaf traits are sometimes better correlated with growth and mortality rates, particularly in closedcanopy forests (Reich et al. 1992, 2003, Poorter and Bongers 2006). Leaf life span, leaf tissue density, leaf nitrogen concentration, photosynthetic capacity, and dark respiration rates all contribute to shade tolerance (Grime 1965, Reich et al. 1995, 2003, Lusk and Reich 2000, Poorter and Bongers 2006, Kitajima and Poorter 2010). High $\mathrm{CO}_{2}$ exchange rates are associated with the fast-growth, high-mortality end of the growth-mortality trade-off while low respiratory carbon losses and long leaf lifetimes are associated with the slow-growth, lowmortality end of the trade-off. These "hard" leaf traits are mechanistically linked to carbon economy and might be better predictors of demographic rates than is LMA.

\section{Seed mass}

Relationships between SM and demographic rates were weak or inconsistent. Seed mass-growth relationships were significant but with coefficients of determination of just $6-9 \%$ (Tables 1 and 2). The SM-mortality relationships were highly variable. The SM-mortality relationship for large BCI trees was relatively strong in Poorter et al. (2008) and insignificant in this study (Table 2). Visual inspection of Fig. 2 in Poorter et al. (2008) suggests their BCI seed size-mortality relationship depends on three outliers with minute seeds and high mortality. The sapling SM- $M_{25}$ relationship was relatively strong for species-level values but insignificant for phylogenetically independent contrasts (Tables 1 and 2). There is no evidence for coordinated evolutionary changes in SM and sapling mortality.

Weak and inconsistent relationships between SM and demographic rates are perhaps not surprising given the limited role of seeds at sapling and later life stages. There is a strong positive relationship between SM and the survival of seeds and first-year seedlings on BCI (King et al. 2006b); however, as seedlings mature and seed reserves are depleted, the seed no longer has any direct impact on performance. Subsequent effects of SM on performance must be indirect rather than causal and might be mediated by multi-trait trade-offs that involve SM (Shipley and Peters 1990, Westoby et al. 2002, Wright et al. 2004, Poorter et al. 2006).

Seed mass also illustrates interactions among tradeoffs that affect performance. Consider the well-established seed number-seed mass trade-off in which many small seeds enable colonization ability while fewer larger seeds enable survival under a variety of hazards (Westoby et al. 2002, Muller-Landau 2010). This trade-off rests on the irrefutable but incomplete logic that seed number per gram of seed produced cannot be increased without a decrease in individual seed mass. This logic is incomplete because allocation to reproduction is not limited to seeds. In addition to seeds, reproduction involves pedicels to enhance flower and fruit displays; flowers, nectar, and pollen to enhance outcrossing; and exocarps, mesocarps, and endocarps to protect seeds and enhance dispersal. The seed number-seed mass trade-off is embedded in a large number of potential trade-offs that could compromise subsequent seed massperformance relationships. As just one example, increased allocation to flowers might entail reduced allocation to seeds and smaller seed size but enhance outcrossing so that lifelong performance was actually greater for smaller but outcrossed seeds. Relationships 
between SM and performance are contingent on both ontogenetic stage and values of other traits.

\section{Maximum plant size}

The weakest trait-demographic rate relationships were for $H_{\max }$. All mortality rates were independent of $H_{\text {max }}\left(r^{2} \leq 0.026\right.$; Tables 1 and 2$)$. Maximum height was also the weakest predictor of growth rates of saplings (Table 1); however, $H_{\max }$ was the strongest predictor of growth rates of large trees (Table 2). Poorter et al. (2008) speculated that the $H_{\max }-\mathrm{RGR}$ relationship among large trees might depend on the inclusion of small species whose maximum sizes are not much larger than the lower $10-\mathrm{cm}$ dbh threshold used to define large trees. There are many small tree species on BCI (King et al. 2006b). To explore their contribution to the $H_{\max }-$ RGR relationship, we first included all 67 species with $H_{\text {max }}$ and RGR for large trees $\left(r^{2}=0.28, P<0.001\right.$; Table 2) then 60 of those species with $H_{\max }>15 \mathrm{~m}\left(r^{2}=\right.$ $0.090, P=0.021)$, and finally 49 species with $H_{\max }>20$ $\mathrm{m}\left(r^{2}=0.014, P=0.40\right)$. The $H_{\max }-\mathrm{RGR}$ relationship for large trees became insignificant as a few small tree species were excluded. We conclude that growth and mortality rates are generally independent of $H_{\max }$ on BCI. Bohlman and O'Brien (2006) reached the same conclusion in an earlier study of BCI saplings.

Other studies find stronger $H_{\max }-\mathrm{RGR}$ relationships. This includes significant $H_{\max }-\mathrm{RGR}$ relationships for large trees from three Neotropical forests, which might have been an artifact of the inclusion of small species as discussed in the previous paragraph (Poorter et al. 2008). At the Pasoh Forest Reserve, Malaysia, $H_{\max }$ and RGR are positively correlated within five speciose genera (Thomas 1996), and a dbh-based index of maximum size is positively correlated with sapling RGR in a community-wide analysis but with an $r^{2}$ value of just 0.18 (King et al. 2006a). In sum, the evidence for correlations between $H_{\max }$ and growth rates is mixed. This does not mean that $H_{\max }$ is unimportant. Rather, several other traits tend to covary with $H_{\max }$ and the strongest correlations between $H_{\max }$ and growth rates are contingent on values of other traits (Thomas 1996, Poorter et al. 2006).

\section{Conclusions}

We have established that on BCI (1) there is a strong trade-off between rapid growth under favorable conditions and low mortality under unfavorable conditions for saplings (Fig. 1) but not large trees; (2) wood density explains a significant and substantial proportion of interspecific variation in position along the sapling growth-mortality trade-off (Table 1); and (3) after wood density is incorporated, three other supposedly key functional traits (seed mass, leaf mass per area, and maximum height) add little to the explained variation (Table 1). A wide range of seed masses, maximum heights, and leaf mass per area characterizes species at all positions along the growth-mortality trade-off (Figs. 2 and 3 ).

This does not mean that seed mass, leaf mass per area, and maximum height are unimportant. The saplings evaluated here have already passed through several life history stages including seed arrival, germination, establishment, and growth and survival as seedlings. An obvious next step will be to evaluate traitdemography relationships at these early life stages (Kitajima 1994, Reich et al. 2003, King et al. 2006b, Muller-Landau et al. 2008).

Another important step will incorporate microhabitatdependent performance. Ecological filters at the germination, establishment, and seedling stages restrict saplings to species-specific subsets of the available microhabitats. As an example, low light levels exclude most species from some proportion of potential regeneration sites with the proportion increasing from shade-tolerant to lightdemanding species (Rüger et al. 2009). We only observed saplings of light-demanding species in relatively bright microhabitats. An analysis that controlled variation in light availability might yet detect stronger trait-performance relationships than observed here.

The search for traits that provide additional predictive ability at each life stage should also be expanded. Traits that are well correlated with performance in common garden experiments on young plants might not be correlated with performance at later life stages in real forests. We might need to return to the chemical, physiological, and allometric traits that are mechanistically linked to performance. We might also need to consider the possibility that relationship between any one trait and performance is contingent on values of other possibly unidentified traits (Marks and Lechowicz 2006).

We end with a philosophical question. How much variation do simple traits need to explain to serve as a useful foundation for building conceptual and quantitative models of ecological strategies? If traits explain $20 \%, 30 \%$, or $50 \%$ of all variation in important specieslevel performance metrics, is the glass partially full or partially empty? Perhaps the answer is both. A simple trait-based approach can provide a meaningful first step toward a more predictive and quantitative science that can be further advanced by consideration of other more complex traits as well as ecological factors outside the plant trait envelope.

\section{ACKNOWLEDGMENTS}

Rolando Perez, Salomon Aguilar, and Javier Ballesteros determined wood densities. The F. H. Levinson Fund supported plant trait measurements. The National Science and MacArthur Foundations supported 50-ha plot censuses.

\section{Literature Cited}

Alvarez-Clare, S., and K. Kitajima. 2009. Susceptibility of tree seedlings to biotic and abiotic hazards in the understory of a moist tropical forest in Panama. Biotropica 41:47-56.

Belsley, D. A., E. Kuh, and R. E. Welsch. 1980. Regression diagnostics: identifying influential data and sources of 
collinearity. John Wiley and Sons, New York, New York, USA.

Bohlman, S., and S. O'Brien. 2006. Allometry, adult stature and regeneration requirement of 65 tree species on Barro Colorado Island, Panama. Journal of Tropical Ecology 22: 123-136.

Chave, J., D. Coomes, S. Jansen, S. L. Lewis, N. G. Swenson, and A. E. Zanne. 2009. Towards a worldwide wood economics spectrum. Ecology Letters 12:351-366.

Condit, R., et al. 2006. The importance of demographic niches to tree diversity. Science 313:98-101.

Cornelissen, J. H. C., et al. 2003. Handbook of protocols for standardised and easy measurement of plant functional traits worldwide. Australian Journal of Botany 51:335-380.

Díaz, S., et al. 2004. The plant traits that drive ecosystems: evidence from three continents. Journal of Vegetation Science 15:295-304.

Felsenstein, J. 1985. Phylogenies and the comparative method. American Naturalist 125:1-15.

Garland, T., P. H. Harvey, and A. R. Ives. 1992. Procedures for the analysis of comparative data using phylogenetically independent contrasts. Systematic Biology 41:18-32.

Grime, J. P. 1965. Shade tolerance in flowering plants. Nature 208:161-163.

Grime, J. P., et al. 1997. Integrated screening validates primary axes of specialisation in plants. Oikos 79:259-281.

Grubb, P. J. 1977. The maintenance of species-richness in plant communities: the importance of the regeneration niche. Biological Review 52:107-145.

Hodgson, J. G., P. J. Wilson, R. Hunt, J. P. Grime, and K. Thompson. 1999. Allocating C-S-R plant functional types: a soft approach to a hard problem. Oikos 85:282-294.

Hubbell, S. P., and R. B. Foster. 1992. Short-term dynamics of a neotropical forest: why ecological research matters to tropical conservation and management. Oikos 63:48-61.

King, D. A., S. J. Davies, and M. N. Nur Sapardi. $2006 a$. Growth and mortality are related to adult tree size in a Malaysian mixed dipterocarp forest. Forest Ecology and Management 223:152-158.

King, D. A., S. J. Wright, and J. H. Connell. 2006b. The distribution of maximum tree heights in tropical vs. temperate forests: Cause or consequence of tropical diversity? Journal of Tropical Ecology 22:11-24.

Kitajima, K. 1994. Relative importance of photosynthetic and allocation traits as correlates of seedling shade tolerance of 15 tropical tree species. Oecologia 98:419-428.

Kitajima, K., and L. Poorter. 2008. Functional basis for resource niche partitioning by tropical trees. Pages 172-188 in W. P. Carson and S. A. Schnitzer, editors. Tropical forest community ecology. Blackwell, Oxford, UK.

Kitajima, K., and L. Poorter. 2010. Tissue-level leaf toughness, but not lamina thickness, predicts sapling leaf lifespan and shade tolerance of tropical tree species. New Phytologist, in press. [doi: 10.1111/j.1469-8137.2010.03212.x]

Kress, W. J., et al. 2009. Plant DNA barcodes and a community phylogeny of a tropical forest dynamics plot in Panama. Proceedings of the National Academy of Sciences USA 106: $18627-18632$

Lambers, H., and H. Poorter. 1992. Inherent variation in growth rate between higher plants: a search for physiological causes and ecological consequences. Advances in Ecological Research 23:187-261.

Lavorel, S., and E. Garnier. 2002. Predicting changes in community composition and ecosystem functioning from plant traits: revisiting the Holy Grail. Functional Ecology 16: 545-556.

Lusk, C. H., and P. B. Reich. 2000. Relationships of leaf dark respiration with light environment and tissue nitrogen content in juveniles of 11 cold-temperate tree species. Oecologia 123:318-329.
Marks, C. O., and M. J. Lechowicz. 2006. Alternative designs and the evolution of functional diversity. American Naturalist 167:55-66.

McGill, B. J., B. J. Enquist, E. Weiher, and M. Westoby. 2006. Rebuilding community ecology from functional traits. Trends in Ecology and Evolution 21:178-185.

Muller-Landau, H. C. 2004. Interspecific and inter-site variation in wood specific gravity of tropical trees. Biotropica 36 : $20-32$.

Muller-Landau, H. C. 2010. The tolerance-fecundity tradeoff and the maintenance of diversity in seed size. Proceedings of the National Academy of Sciences USA, in press.

Muller-Landau, H. C., S. J. Wright, O. Calderon, R. Condit, and S. P. Hubbell. 2008. Interspecific variation in primary seed dispersal in a tropical forest. Journal of Ecology 96:653667.

Pacala, S. W., C. D. Canham, J. Saponara, J. A. Silander, R. K. Kobe, and E. Ribbens. 1996. Forest models defined by field measurements: estimation, error analysis and dynamics. Ecological Monographs 66:1-43.

Pagel, M. D. 1992. A method for the analysis of comparative data. Journal of Theoretical Biology 156:431-442.

Patiño, S., et al. 2009. Branch xylem density variations across the Amazon Basin. Biogeosciences 6:545-568.

Poorter, H., Ü. Niinemets, L. Poorter, I. J. Wright, and R. Villar. 2009. Causes and consequences of variation in leaf mass per area (LMA): a meta-analysis. New Phytologist 182: 565-588.

Poorter, L. 2007. Are species adapted to their regeneration niche, adult niche or both? American Naturalist 169:433442.

Poorter, L., and F. Bongers. 2006. Leaf traits are good predictors of plant performance across 53 rain forest species. Ecology 87:1733-1743.

Poorter, L., L. Bongers, and F. Bongers. 2006. Architecture of 54 moist forest tree species: traits, trade-offs, and functional groups. Ecology 87:1289-1301.

Poorter, L., et al. 2008. Are functional traits good predictors of demographic rates? Evidence from five Neotropical forests. Ecology 89:1908-1920.

Reich, P. B., D. S. Ellsworth, and C. Uhl. 1995. Leaf carbon and nutrient assimilation and conservation in species of differing successional status in an oligotrophic Amazonian forest. Functional Ecology 9:65-76.

Reich, P. B., M. B. Walters, and D. S. Ellsworth. 1992. Leaf lifespan in relation to leaf, plant and stand characteristics among diverse ecosystems. Ecological Monographs 62:365392.

Reich, P. B., I. Wright, J. Cavender-Bares, J. Craine, J. Oleksyn, M. Westoby, and M. B. Walters. 2003. The evolution of plant functional variation: traits, spectra, and strategies. International Journal of Plant Sciences 164:S143S164.

Rüger, N., A. Huth, S. P. Hubbell, and R. Condit. 2009. Response of recruitment to light availability across a tropical lowland rain forest community. Journal of Ecology 97:1360 1368 .

Shipley, B., and R. H. Peters. 1990. The allometry of seed weight and seedling relative growth rate. Functional Ecology 4:523-539.

Thomas, S. C. 1996. Asymptotic height as a predictor of growth and allometric characteristics in Malaysian rain forest trees. American Journal of Botany 83:556-566.

Webb, C. O., D. D. Ackerly, and S. W. Kembel. 2008. Phylocom: software for the analysis of phylogenetic community structure and trait evolution. Bioinformatics 24:20982100.

Weiher, E., A. van der Werf, K. Thompson, M. Roderick, E. Garnier, and O. Eriksson. 1999. Challenging Theophrastus: a common core list of plant traits for functional ecology. Journal of Vegetation Science 10:609-620. 
Westoby, M., D. S. Falster, A. T. Moles, P. A. Vesk, and I. J. Wright. 2002. Plant ecological strategies: some leading dimensions of variation between species. Annual Review of Ecology and Systematics 33:125-159.

Westoby, M., and I. J. Wright. 2006. Land-plant ecology on the basis of functional traits. Trends in Ecology and Evolution 21:261-268.

Wright, I. J., et al. 2004. The worldwide leaf economics spectrum. Nature 428:821-827.
Wright, I. J., et al. 2007. Relationships among key dimensions of plant trait variation in seven Neotropical forests. Annals of Botany 99:1003-1015.

Wright, S. J., H. C. Muller-Landau, R. Condit, and S. P. Hubbell. 2003. Gap-dependent recruitment, realized vital rates, and size distributions of tropical trees. Ecology 84: $3174-3185$.

Yoda, K. 1974. Three-dimensional distribution of light intensity in a tropical rain forest of West Malaysia. Japanese Journal of Ecology 24:247-254.

\section{SUPPLEMENT}

All data used in analyses of relationships among functional traits and demographic rates for trees and saplings from Barro Colorado Island, Panama (Ecological Archives E091-257-S1). 